\title{
Transition Time to Full Oral Feeding Skill and Its Determinants in Very Preterm Infants: A Single Center Experience
}

\author{
๑ilkay Err1, ๑ Ayla Günlemez² \\ 1University of Health Sciences Turkey, Kocaeli Derince Training and Research Hospital Clinic of, Neonatology, Kocaeli, Turkey \\ 2Kocaeli University Faculty of Medicine, Department of Pediatrics, Division of Neonatology, Kocaeli, Turkey
}

\begin{abstract}
Aim: Transition from tube to full oral feeding (FOF) represents an important milestone for very preterm infants, and can be affected by various factors. This study aimed to evaluate transition time to FOF in very preterm infants and to identify the factors affecting this ability.

Materials and Methods: In this 4-year study, infants' characteristics, feeding steps, and comorbidities were retrospectively evaluated. Infants were categorized into two groups based on FOF timing and comparisons were made. Logistic regression analysis was used to examine any affecting factors.

Results: Transition from tube to FOF occurred on a median of 20 days. There were 48 and 52 neonates in the $\leq 20$ days and >20 days groups, respectively. Gender, delivery type, and antenatal history were similar between the groups. The majority were supported with formula. More infants were at $>30$ weeks of gestational age, and had a birth weight $>1.47 \mathrm{~kg}$ in the $\leq 20$ days group. A lesser proportion of these required invasive interventions, and had comorbidities. In this study, the requirement of non-invasive ventilation $\leq 3$ days, receiving kangaroo mother care, the promotion of non-nutritive sucking within the first week, and the achievement of full enteral feeding in $\leq 14$ days were associated with a positive effect on the transition to FOF in $\leq 20$ days. However, a gestational age of $\leq 30$ weeks and diagnosis of bronchopulmonary dysplasia had a negative effect.
\end{abstract}

Conclusion: This was one of the few studies to investigate the timing of transition to FOF in very preterm infants as well as its affecting factors. Further studies are required to provide guidance on interventions to shorten FOF time and to provide kangaroo mother care, non-nutritive sucking and breastfeeding in the very preterm population.

Keywords: Very preterm infants, full oral feeding, affecting factors

\section{Introduction}

Survival in very preterm (VPT) infants born at $<32$ gestational weeks has improved significantly owing to advances in perinatal interventions and neonatal intensive care practices which could have led to airway and feeding morbidities (1). Transition from tube to full oral feeding
(FOF), which is a major discharge criterion indicating the maturity and health of the preterm, represents an essential milestone in the feeding process for these infants $(2,3)$.

Swallowing is already present in the fetus by week 16 of gestation and is expected to be functional at around 34-36 gestational weeks. VPT infants usually experience

\section{Address for Correspondence}

Ilkay Er, University of Health Sciences Turkey, Kocaeli Derince Training and Research Hospital Clinic of, Neonatology, Kocaeli, Turkey Phone: +90 5306911517 E-mail: ilkayer7778@yahoo.com ORCID: orcid.org/0000-0003-4562-3829 Received: 17.03.2021 Accepted: 05.05.2021

${ }^{\circ}$ Copyright 2021 by Ege University Faculty of Medicine, Department of Pediatrics and Ege Children's Foundation The Journal of Pediatric Research, published by Galenos Publishing House. 
difficulties in coordinating sucking, breathing, and swallowing, and thus are tube-fed for some duration $(4,5)$. However, the developmental stages of oral feeding skills have not been very well characterized in prematurely born infants $(6,7)$. Furthermore, the feeding process may be influenced by a number of factors including the infants' own characteristics, receiving kangaroo mother care (KMC), and the promotion of non-nutritive sucking (NNS), as well as by the presence of neonatal comorbidities such as hemodynamically significant patent ductus arteriosus (hsPDA), necrotizing enterocolitis (NEC), infections, and bronchopulmonary dysplasia (BPD) $(6,8)$. It is known that nutrition is a crucial component of care in VPT infants. Feeding difficulties leading to inadequate nutrition during this critical period have been associated with the duration of hospital stay as well as growth retardation, and adverse neurodevelopmental outcomes in the later stages of life (9).

In this retrospective study, we aimed to evaluate the time to FOF and identify the factors affecting transition to FOF in preterm infants born at $<32$ weeks of gestation and followed up in the neonatal intensive care unit (NICU) of our hospital.

\section{Materials and Methods}

\section{Study Design}

In this study involving infants born at $<32$ weeks of gestation between January 2016 and December 2019 at Kocaeli Derince Training and Research Hospital, the following data retrieved from the medical records were retrospectively evaluated: Perinatal, demographic and clinical characteristics including maternal age, maternal disease, antenatal steroid use, delivery mode, APGAR score, clinical risk index-II score (CRIP-II score predicting neonatal mortality at admission), sex, gestational age (GA), birth weight (BW), percentile for GA on the Fenton curve (10), surfactant treatment, duration of intubation/non-invasive ventilation (NIV)/oxygen support, caffeine use, duration of hospitalization; feeding steps; and comorbidities. Infants with malformations of the head, neck, and gastrointestinal (GI) tract; genetic abnormalities; and $\mathrm{BW}$ of $<10^{\text {th }}$ percentile for gestation on the Fenton curve were excluded. The study protocol was approved by the University of Health Sciences Turkey, Kocaeli Derince Training and Research Hospital, Clinical Research ethics committee (no: 2020/72).

The feeding steps defined in study were as follows: In addition to parenteral nutrition (PN), colostrum was started on the first day of life directly by mouth or through orogastric tube. After minimal enteral feeding (EF) for 3-5 days, EF was increased in accordance with GA/BW by increments of either $20 \mathrm{~mL} / \mathrm{kg} /$ day or $30 \mathrm{~mL} / \mathrm{kg} /$ day at 2-3 hour intervals. Feedings consisted of either fortified mother's milk or preterm formula. Fortification was initiated at an EF dosage of $50 \mathrm{~mL} / \mathrm{kg} /$ day. When cardiorespiratory stability was attained, even if it was with NIV support, KMC and NNS (via mostly an expressed breast or via a suitable pacifier) were initiated. While PN was gradually reduced, progression to full EF (FEF) was carried out based on GI tolerance. At this stage, infants were transitioned to oral feeding, when they exhibited oral-motor cues and coordinated sucking, breathing, and swallowing. The tube was removed when the infant could receive more than $80-85 \%$ of FEF via breast or bottle. Target weight gain was set at a rate of $15-20 \mathrm{~g} / \mathrm{kg} /$ day $(8,11,12)$. Once FOF was achieved, ad libitum feeding was started before discharge. Time to FEF and transition to FOF were recorded for each infant. FEF was defined as an enteral volume of at least $150 \mathrm{cc} / \mathrm{kg} /$ day containing 120 calories/kg/ day by gavage feeding alone $(8,11)$.

Feeding intolerance was defined as the presence of one or more of the following: a) vomiting more than 3 times in any 24-hour period; b) any episode of bile-stained vomiting; c) abdominal wall erythema/tenderness and/or decreased bowel sounds; d) gross/occult blood in stools; e) milky gastric residue $>50 \%$ of the previous feed volume (residue was checked only in cases of clinical suspicion). These criteria for feeding intolerance also comprised indications for temporary cessation of feeds $(8,13,14)$.

Co-morbid factors identified in the study population included hsPDA, NEC, late-onset sepsis, and BPD. hsPDA was defined as a ductus arteriosus diameter of $\geq 1.5 \mathrm{~mm}$ and/or a left atrium/aortic root ratio $\geq 1.5$, as assessed by Doppler echocardiography within the first 48-72 hours of life (15). NEC was suspected in infants with abdominal/ systemic symptoms and signs, and they were categorized using modified Bell's classification (14). Sepsis with and without positive blood cultures after 72 hours of life was defined as late-onset culture proven and clinical sepsis, respectively (16). BPD was defined as the requirement for more than 28 days of supplemental oxygen between birth and 36 weeks of corrected GA (17).

\section{Statistical Analysis}

Statistical analyses were performed using IBM-SPSS for Windows version 20.0 (IBM Corp., Armonk, NY, USA). Kolmogorov-Smirnov test was used to test the normality of the data distributions. Continuous variables were expressed as mean ( \pm standard deviation) and median $\left(25^{\text {th }}-75^{\text {th }}\right.$ percentiles). Categorical variables were expressed as counts 
(percentages) or percentages. Intergroup comparisons of normally and non-normally distributed continuous variables were performed using Student's t-test and Mann-Whitney $U$ test, respectively. Yates' chi-square and Monte Carlo chi-square tests were used for intergroup comparisons of categorical variables. A two-sided $p$-value of $<0.05$ was considered statistically significant.

To investigate the variables that had an effect on feeding in VPT infants, median time of FOF was chosen as the cutoff point to divide the participants into comparable groups, in line with the published literature $(18,19)$. For other factors, mean/median values were used to compose the subgroups. Subsequently, binary logistic regression analysis was used to determine those variables with a significant effect on feeding status.

\section{Results}

\section{Baseline Characteristics of the VPT Infants}

A total of 100 infants (53 males) born at $<32$ weeks of gestation who were admitted to the NICU and met the inclusion criteria were included in this 4-year study. The GA and BW of the infants were 30 (28.4-31) weeks and 1.47 \pm 0.34 $\mathrm{kg}$, respectively. Transition from FEF to FOF occurred on day 20 (10-35), at 32.6 (32.4-33.5) postnatal weeks. The infants were discharged at 34.5 (32.5-37.2) weeks of age, with a weight of $2.01 \pm 0.20 \mathrm{~kg}$, and all of them were on ad libitum feeding. The baseline characteristics of the study population are presented in Table I. The majority were supported with formula during hospitalization. The percentage of those exclusively fed with mother's milk (via breast or bottle) was $16 \%$ at discharge.

\begin{tabular}{|c|c|}
\hline Female/male, \% & $47 / 53$ \\
\hline Vaginal delivery/cesarean, $\%$ & $31 / 69$ \\
\hline Birth weight $(\mathrm{kg})$, mean $\pm \mathrm{SD}$ & $1.47 \pm 0.34$ (minimum 0.65 - maximum 1.85 ) \\
\hline Gestational age, median $\left(25^{\text {th }}-75^{\text {th }}\right)$ & 30 (28.4-31) (minimum 25 - maximum 31.4) \\
\hline Maternal age $(y r s)$, median $\left(25^{\text {th }}-75^{\text {th }}\right)$ & $28(22-34)$ \\
\hline Ratio of maternal PPROM*, \% & 61 \\
\hline Ratio of antenatal steroid, \% & $42(42)$ \\
\hline APGAR score at 5 min, median $\left(25^{\text {th }}-75^{\text {th }}\right)$ & $8(7-9)$ \\
\hline CRIP\& score, median $\left(25^{\text {th }}-75^{\text {th }}\right)$ & $6(2-8)$ \\
\hline Ratio of surfactant administration, $\%$ & $69(69)$ \\
\hline Duration of intubation (days), median $\left(25^{\text {th }}-75^{\text {th }}\right)$ & $0(0-2)$ \\
\hline Duration of NIV support (days), median $\left(25^{\text {th }}-75^{\text {th }}\right)$ & $3(1-8)$ \\
\hline Ratio of caffeine receivement, $\%$ & $83(83)$ \\
\hline Day of first KMC and NNS, median $\left(25^{\text {th }}-75^{\text {th }}\right)$ & $7.5(4-20)$ \\
\hline Duration of using PN ${ }^{ \pm}$(days), median $\left(25^{\text {th }}-75^{\text {th }}\right)$ & $10(5-14)$ \\
\hline Day of achieve to FEF ${ }^{\beta}$ (days), median $\left(25^{\text {th }}-75^{\text {th }}\right)$ & $14(10-18)$ \\
\hline Day of transition to FOF ${ }^{€}$ (days), median $\left(25^{\text {th }}-75^{\text {th }}\right)$ & $20(10-35)$ \\
\hline Number of epizodes of feeding cessation, median $\left(25^{\text {th }}-75^{\text {th }}\right)$ & $3(0-5)$ \\
\hline Ratio of hsPDA $\$, \%$ & 16 \\
\hline Ratio of $\mathrm{NEC}^{\mathrm{K}} \pm$ clinical/proven sepsis, \% & 10 \\
\hline Ratio of late-onset neonatal sepsis (clinical/proven), \% & $28 / 22$ \\
\hline Ratio of $\mathrm{BPD}^{\mathrm{F}}$ diagnosing (mild/moderate), \% & $19 / 9$ \\
\hline Ratio of abnormal cranial USC findings, $\%$ & 15 \\
\hline Duration of hospitalization (days), median $\left(25^{\text {th }}-75^{\text {th }}\right)$ & $30(16.3-47.8)$ \\
\hline Weight at discharge $(\mathrm{Kg})$, mean $\pm \mathrm{SD}$ & $2.01 \pm 0.20$ \\
\hline Postnatal weeks at discharge, median $\left(25^{\text {th }}-75^{\text {th }}\right)$ & $34.5(32.5-37.2)$ \\
\hline \multicolumn{2}{|c|}{$\begin{array}{l}\text { *: Preterm premature rupture of membrane, }, \text { : Non-invasive ventilation, } \infty: \text { Kangaroo mother care and non-nutritive sucking, \&: Clinical Risk index II score, } £ \text { : Parenteral } \\
\text { nutrition, } \beta \text { : Full enteral feeding, €: Full oral feeding, S: Hemodynamically significant patent ductus arteriosus, K: Necrotizing enterocolitis, F: Bronchopulmonary } \\
\text { dysplasia, SD: Standard deviation, APGAR: Appearance, pulse, grimace, activity, and respiration, PPROM: Preterm premature rupture of membrane, CRIP: Clinical risk } \\
\text { index, NIV: Non-invasive ventilation, KMC: Kangaroo mother care, NNS: Non-nutritive sucking, PN: Parenteral nutrition, FEF: Full enteral feeding, FOF: Full oral feeding, } \\
\text { hsPDA: Hemodynamically significant patent ductus arteriosus, NEC: Necrotizing enterocolitis, BPD: Bronchopulmonary dysplasia, USG: Ultrasonography }\end{array}$} \\
\hline
\end{tabular}




\section{Comparison of Neonates with FOF $\leq \mathbf{2 0}$ and $>\mathbf{2 0}$ Days}

There were 48 and 52 neonates with a GA of 31 (30.431.2) weeks and 28 (27-29.3) weeks, respectively, in the two study groups defined on the basis of a median time to FOF of $\leq 20$ days and $>20$ days, respectively.

In the comparison of both groups, more infants in the FOF $\leq 20$ days group were $>30$ weeks of GA, had BW $>1.47 \mathrm{~kg}$, and had a CRIP score $\leq 6$. A smaller proportion of those in this FOF $\leq 20$ days group received surfactant, were supported with NIV $>3$ days, needed caffeine/PN; and a higher proportion received $\mathrm{KMC}$ and were provided with NNS within 7.5 days of life. Additionally, time to FOF of $\leq 20$ days was associated with no or reduced hsPDA + NEC+/-sepsis (all cases with NEC had stage 1-2 disease), a lesser occurrence of mild/moderate BPD, having no or $\leq 3$ episodes of feeding cessation, an absence of abnormal cranial ultrasound findings at admission, and higher rates of FEF $\leq 14$ days and discharge within 30 days. The groups were similar in terms of gender, delivery type, antenatal and maternal history, and APGAR score distribution ( $p>0.05$ ). Table II shows the comparison of these two groups.

Table II. The comparison of $\leq 20$ days and $>20$ days FOF groups, $n$ (\%)

\begin{tabular}{|c|c|c|c|}
\hline & $\leq 20$ days FOF group, $(n=48)$ & $>20$ days FOF group, $(n=52)$ & $p$-value ${ }^{*}$ \\
\hline $\begin{array}{l}\text { Gender } \\
\text { Female } \\
\text { Male }\end{array}$ & $\begin{array}{l}26(56.2) \\
22(45.8)\end{array}$ & $\begin{array}{l}21(40.4) \\
31(59.6)\end{array}$ & 0.238 \\
\hline $\begin{array}{l}\text { Style of delivery } \\
\text { Vaginal } \\
\text { Cesarean }\end{array}$ & $\begin{array}{l}14(24.2) \\
34(70.8)\end{array}$ & $\begin{array}{l}17(32.7) \\
35(67.3)\end{array}$ & 0.869 \\
\hline $\begin{array}{l}\text { Birth weight (kg) } \\
\leq 1.47 \mathrm{~kg} \\
>1.47 \mathrm{~kg}\end{array}$ & $\begin{array}{l}10(20.8) \\
38(79.2)\end{array}$ & $\begin{array}{l}39(75) \\
13(25)\end{array}$ & $<0.001$ \\
\hline $\begin{array}{l}\text { Gestational age } \\
\leq 30 \text { weeks } \\
>30 \text { weeks }\end{array}$ & $\begin{array}{l}12(25) \\
36(75)\end{array}$ & $\begin{array}{l}44(84.6) \\
8(15.4)\end{array}$ & $<0.001$ \\
\hline $\begin{array}{l}\text { Maternal age } \\
\leq 28 \text { years } \\
>28 \text { years }\end{array}$ & $\begin{array}{l}24(50) \\
24(50)\end{array}$ & $\begin{array}{l}24(46.2) \\
28(53.8)\end{array}$ & 0.854 \\
\hline $\begin{array}{l}\text { Maternal disease } \\
\text { PPROM* }^{*} \\
\text { Preeclampsia, others }\end{array}$ & $\begin{array}{l}26(54.2) \\
22(45.8)\end{array}$ & $\begin{array}{l}35(67.3) \\
17(32.7)\end{array}$ & 0.254 \\
\hline $\begin{array}{l}\text { APGAR score at } 5 \text { minute } \\
\leq 8 \\
>8\end{array}$ & $\begin{array}{l}9(18.8) \\
39(81.2)\end{array}$ & $\begin{array}{l}11(21.2) \\
41(78.8)\end{array}$ & 0.960 \\
\hline $\begin{array}{l}\text { CRIB }^{\&} \text { score } \\
\leq 6 \\
>6\end{array}$ & $\begin{array}{l}38(79.2) \\
10(20.8)\end{array}$ & $\begin{array}{l}14(26.9) \\
38(73.1)\end{array}$ & $<0.001$ \\
\hline $\begin{array}{l}\text { Antenatal steroid } \\
\text { None/incomplete } \\
\text { Completed }\end{array}$ & $\begin{array}{l}29(60.4) \\
19(39.6)\end{array}$ & $\begin{array}{l}29(55.8) \\
23(44.2)\end{array}$ & 0.789 \\
\hline $\begin{array}{l}\text { Surfactant administration } \\
\text { Not administered } \\
\text { Via INSURE } \\
\text { Remaining intubated }\end{array}$ & $\begin{array}{l}24(50) \\
10(20.8) \\
14(29.2)\end{array}$ & $\begin{array}{l}7(13.5) \\
15(28.8) \\
30(57.7)\end{array}$ & $<0.001$ \\
\hline $\begin{array}{l}\text { Duration of NIV' support } \\
\leq 3 \text { days } \\
>3 \text { days }\end{array}$ & $\begin{array}{l}43(89.6) \\
5(10.4)\end{array}$ & $\begin{array}{l}16(30.8) \\
36(69.2)\end{array}$ & $<0.001$ \\
\hline $\begin{array}{l}\text { Caffeine } \\
\text { Not received } \\
\text { Received }\end{array}$ & $\begin{array}{l}17(35.4) \\
31(67.6)\end{array}$ & $\begin{array}{l}0(0) \\
52(100)\end{array}$ & $<0.001$ \\
\hline $\begin{array}{l}\text { Receiving } \text { KMC\&NNS }^{\infty} \\
\leq 7.5 \text { days } \\
>7.5 \text { days }\end{array}$ & $\begin{array}{l}39(81.2) \\
9(18.8)\end{array}$ & $\begin{array}{l}10(19.2) \\
42(80.8)\end{array}$ & $<0.001$ \\
\hline
\end{tabular}


Table II. continued

\begin{tabular}{|c|c|c|c|}
\hline $\begin{array}{l}\text { Duration of PN } \mathbf{P N}^{\mathbf{E}} \\
\leq 10 \text { days } \\
>10 \text { days }\end{array}$ & $\begin{array}{l}39(81.2) \\
9(18.8)\end{array}$ & $\begin{array}{l}7(13.5) \\
45(86.5) \\
\end{array}$ & $<0.001$ \\
\hline $\begin{array}{l}\text { Achieving FEF } \\
\leq 14 \text { days } \\
>14 \text { days }\end{array}$ & $\begin{array}{l}43(89.6) \\
5(10.4)\end{array}$ & $\begin{array}{l}7(13.5) \\
45(86.5)\end{array}$ & $<0.001$ \\
\hline $\begin{array}{l}\text { Number of episodes of feeding cessation } \\
\text { None }-\leq 3 \\
>3\end{array}$ & $\begin{array}{l}41(85.4) \\
7(14.6)\end{array}$ & $\begin{array}{l}13(25) \\
39(75)\end{array}$ & $<0.001$ \\
\hline $\begin{array}{l}\text { Comorbidities } \\
\text { None } \\
\text { hsPDA }^{\$}+\mathrm{NEC}^{\mathrm{K}} \pm \text { sepsis } \\
\text { Sepsis (clinical/proven) }\end{array}$ & $\begin{array}{l}24(50) \\
5(10.4) \\
19(39.6)\end{array}$ & $\begin{array}{l}0(0) \\
21(40.4) \\
31(59.6)\end{array}$ & $<0.001$ \\
\hline $\begin{array}{l}\text { BPD } \\
\text { Mild/Moderate } \\
\text { None }\end{array}$ & \begin{tabular}{|l}
$4(8.3)$ \\
$44(91.7)$
\end{tabular} & $\begin{array}{l}24(46.2) \\
28(53.8)\end{array}$ & $<0.001$ \\
\hline $\begin{array}{l}\text { Cranial ultrasound findings at admission } \\
\text { Normal } \\
\text { Abnornal }^{\varnothing}\end{array}$ & $\begin{array}{l}48(100) \\
0(0)\end{array}$ & \begin{tabular}{|l}
$37(71.2)$ \\
$15(28.8)$ \\
\end{tabular} & $<0.001$ \\
\hline $\begin{array}{l}\text { Duration of hospitalization } \\
\leq 30 \text { days } \\
>30 \text { days }\end{array}$ & $\begin{array}{l}37(77.1) \\
11(22.9) \\
\end{array}$ & \begin{tabular}{|l|l|}
$6(11.5)$ \\
$46(88.5)$ \\
\end{tabular} & $<0.001$ \\
\hline \multicolumn{4}{|c|}{ 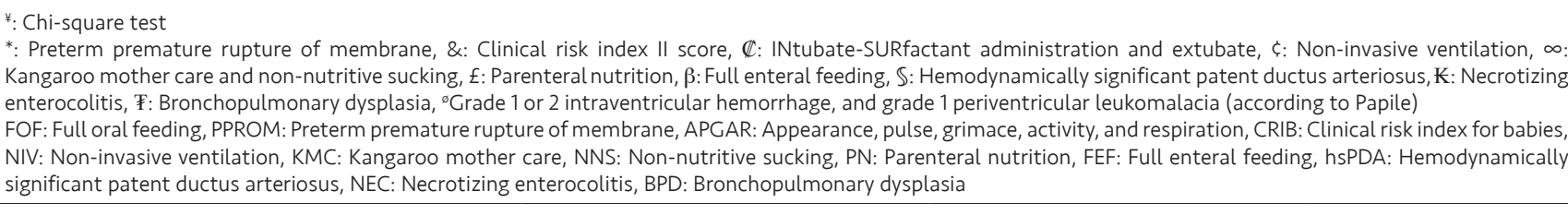 } \\
\hline
\end{tabular}

In the groups of FOF $\leq 20$ days and FOF $>20$ days, the proportions of those fed exclusively mother's milk and exclusively formula were $12 \%$ and $4 \%$ in the former and $2 \%$ and $5 \%$ in the latter. The rest were fed both, but mostly formula fed rather than mother's milk. The main mode of feeding was via bottle in both groups (75\% and $90 \%$ in FOF $\leq 20$ days group and FOF $>20$ days group, respectively).

Logistic Regression Analysis for Factors that Affect the Transition to FOF at $\leq 20$ Days

Based on the results of binary regression analysis, the factors associated with an increased likelihood of transition to FOF in $\leq 20$ days included GA of $>30$ weeks, requirement of NIV support $\leq 3$ days, receiving KMC and the promotion of NNS within the first 7.5 postnatal days, achievement of FEF in $\leq 14$ days, and an absence of BPD (Table III). Other factors were not found to have a significant effect on the transition to FOF ( $p>0.05)$.

\section{Discussion}

Although the prevalence and long-term outcomes of feeding difficulties in premature infants are well-known, the time to attain oral feeding skills as well as the factors that influence this process have not been very well characterized $(6,7,9)$. This study revealed that VPT infants with $>30$ weeks of $\mathrm{GA}, \mathrm{BW}$ of $>1.47 \mathrm{~kg}$, reduced need for interventions, and a lower number of co-morbid conditions were more likely to have time to FOF of $\leq 20$ days. The requirement for NIV support $\leq 3$ days, receiving KMC and the promotion of NNS within the first week, and the ability to achieve FEF in $\leq 14$ days had positive effects on the transition to FOF. Conversely, GA of $\leq 30$ weeks and a diagnosis of BPD prolonged this transition process.

GA, neurodevelopmental maturity level, the quality of care in the NICU, and the facilitation of cue-based oral feeding are among the reported determinants of oral feeding ability in preterm infants (20). When compared to term infants, oral feeding in preterm infants is complicated by innate differences in muscle tone and independent or interdependent suck, swallow and breathe coordination $(2,3,5,21)$. Prematurity can further impair brain development and contribute to poor feeding skills by leading to decreased myelination and white matter disturbances as well as 
Table III. The results of binary logistic regression analysis of factors affecting transition to FOF in $\leq 20$ days

\begin{tabular}{|c|c|c|c|c|c|}
\hline & $\beta$ & SE & Wald & p-value & OR (\%95 Cl) \\
\hline Birth weight of $>1.47 \mathrm{~kg}$ & 0.445 & 0.945 & 0.228 & 0.633 & $1.56(0.25-16.69)$ \\
\hline Gestation age of $>30$ weeks & $-2,364$ & 0.745 & 2,988 & 0.012 & $4.34(0.64-6.66)$ \\
\hline Caffein and surfactant treatment & 18,880 & - & 0.000 & 0.998 & - \\
\hline Requirement to NIV'support $\leq 3$ days & 2,844 & 1,123 & 3,413 & 0.011 & $5.87(1.90-8.51)$ \\
\hline Receiving $\mathrm{KMC}$ and $\mathrm{NNS}^{\infty}$ within first 7.5 days & 2,404 & 0.867 & 3,686 & 0.006 & $7.11(2.02-9.57)$ \\
\hline Achievement of $\mathrm{FEF}^{\beta} \leq 14$ days & 3,138 & 1,042 & 3,075 & 0.003 & $8.10(4.99-10.49)$ \\
\hline Presence of hsPDA ${ }^{\$}+\mathrm{NEC}^{\mathrm{K}}+/$-sepsis & 18,988 & - & 0.000 & 0.998 & - \\
\hline Diagnosis of $\mathrm{BPD}^{\mathrm{F}}$ & $-3,847$ & 1,807 & 4,531 & 0.033 & $11.61(6.66-15.36)$ \\
\hline $\begin{array}{l}\text { No or } \leq 3 \text { episodes of feeding cessation and duration of } \\
\text { PN } \leq 10 \text { days }\end{array}$ & 1,511 & 0.871 & 2,006 & 0.083 & $4.53(0.82-24.99)$ \\
\hline $\begin{array}{l}\text { CRIB }^{\&} \text { score } \leq 6 \text { and absence of abnormal cranial } \\
\text { ultrasound findings }{ }^{\circ} \text { at admission }\end{array}$ & 18,880 & - & 0.000 & 0.998 & - \\
\hline \multicolumn{6}{|c|}{$\begin{array}{l}\text { \&: Clinical risk index II score, } \zeta \text { : Non-invasive ventilation, } \infty \text { : Kangaroo mother care and non-nutritive sucking, £: Parenteral nutrition, } \beta \text { : Full enteral feeding, } \mathbb{S} \text { : } \\
\text { Hemodynamically significant patent ductus arteriosus, K: Necrotizing enterocolitis, F: Bronchopulmonary dysplasia } \\
\text { 'Grade } 1 \text { or } 2 \text { intraventricular hemorrhage, and grade } 1 \text { periventricular leukomalacia (according to Papile) } \\
\text { FOF: Full oral feeding, OR: Odds ratio, SE: It is similar to a standard deviation to a mean, NIV: Non-invasive ventilation, KMC: Kangaroo mother care, NNS: Non-nutritive } \\
\text { sucking, FEF: Full enteral feeding, hsPDA: Hemodynamically significant patent ductus arteriosus, NEC: Necrotizing enterocolitis, BPD: Bronchopulmonary dysplasia, PN: } \\
\text { Parenteral nutrition, CRIB: Clinical risk index for babies }\end{array}$} \\
\hline
\end{tabular}

by delaying the development of a specialized neural circuit known as the suck central pattern generator $(9,22)$. Additionally, maturational delays in Gl motility and/or different comorbidities affect feeding skills during the postnatal period (6). Although a variety of tools to assess the readiness for sucking in pre-terms have been proposed, the 2016 Cochrane meta-analysis suggested a lack of evidence to estimate their effects on the time to establish FOF (7).

To best of our knowledge, only a limited number of previous studies have investigated time to attain oral feeding skills in VPT infants as well as the factors or comorbidities that affect this process $(6,19,23,24)$. In a study by Jadcherla et al. (6) where three strata were defined based on GA at birth (i.e., <28, 28-32 and 32-35 weeks), the impact of prematurity and comorbidities on feeding milestones were extensively explored for the first time. Regardless of GA, most neonates were discharged on FOF at $<37$ weeks' gestation in that study. However, in addition to having prolonged hospitalization, compared with infants $>28$ weeks of GA, those with $<28$ weeks of GA had significant delays in feeding with respect to FEF and FOF. A correlation was also found between postmenstrual age (PMA) of FEF and FOF (6). Similarly, in this study, infants born at $<30$ weeks of $G A$ had significant delays in terms of achieving FEF and FOF, compared to infants born at $>30$ weeks of GA. Furthermore, achieving FEF within 14 days was associated with an 8.1- fold increased likelihood of achieving FOF within 20 days. Time to FOF in our overall study group occurred between 32.4-33.5 postnatal weeks. Patients were discharged at an average 34.5 (32.5-37.2) weeks of age on ad libitum feeding. In Gianni's (23) study, PMA at time of FOF achievement and length of hospital stay in VPT infants were $36.7 \pm 3.68$ weeks and $66.3 \pm 44$ days, respectively. Additionally, in a recent study by Brun et al. (19), time to FOF in VPT infants occurred at 36.6 (35.6-39.2) weeks PMA, and was found to be associated with their duration of hospital stay. The differences in FOF times of these studies may be partially explained by fact that Gianni et al. (23) and Brun et al. (19) also included those infants who were small for GA in their studies.

Lower GA, hypotension, the presence of gastroesophageal reflux, and prolonged ventilation and CPAP were reported to delay time to FOF in Jadcherla's (6) study $(p<0.05)$. Also, each 1-week increment in GA was associated with a 1.25-fold increased chance of achievement of oral feeding. In addition, independent of the presence of comorbidities, apnea, BPD and sepsis were negatively associated with FOF at discharge. According to their findings, a ventilation duration of $>10$ days led to a delay of 1.2 weeks in achieving FOF (6).

In VPT infants, Hwang et al. (24) reported that low BW, moderate-severe BPD, NEC and PDA were predictors for the 
PMA of FOF. Similarly, Giannì et al. (23) reported that low $B W, B P D, C l$ surgery, and having neurosensory disease were independently associated with higher PMA at time of FOF. In the study by Brun et al. (19), duration of NIV and oxygen therapy, BPD, and PDA were associated with an older age at FOF. Moreover, in that study, BPD emerged as the single most important predictor. Consistent with previous reports, we also found that GA $\leq 30$ weeks, the requirement for NIV support $>3$ days, and a diagnosis of BPD were associated with an increased risk of delay for FOF.

Despite some discrepancies between studies, probably due to differences in patient characteristics, management strategies, or analysis methods, BDP appears to be a common risk factor identified in those above-listed studies. Several plausible explanations have been put forward concerning the association of respiratory support with delayed FOF. For instance, infants receiving any type ventilation support could be exposed to nociceptive stimuli in the naso/oro-facial region, which could lead to altered processing of sensory information (25). Additionally, infants are not offered oral feeding mainly due to a fear of aspiration during the treatment of respiratory distress in most NICUs (19). However, BPD and delayed FOF were found to be independently associated with delayed brain maturation at term-equivalent age in a population of VPT infants (26).

In contrast with previous publications, provision of KMC and NNS within the first week and their effects on time to FOF in VPT infants have been specifically evaluated in our study. Preparation of the GI tract with human milk immediately after birth decreases the time required to achieve FEF $(8,11,12)$. At the same time, positive stimulation should be provided by allowing KMC and NNS as often as possible, once the infant has become hemodynamically stable $(3,8,11,12)$. A meta-analysis showed that KMC in low BW infants was associated with reduced mortality, sepsis, hypothermia, length of hospitalization, and increased weight, length, head-circumference, and breastfeeding (27). In a previous study including infants born $<37$ weeks without any comorbidity, BW $>2 \mathrm{~kg}$ as well as receiving $\mathrm{KMC}$ within the first 3 days was associated with 6-fold and 5-fold increases in the likelihood of early initiation of oral feeding, respectively (28).

NNS has been observed during intrauterine life as early as 15 weeks, reported to be rhythmical by 20 weeks, and continues to improve throughout gestation (29). During $\mathrm{KMC}$, a drop of milk may prompt the infant to lick the nipple and then to suck on it over time. Since it provides nutritive suction, NNS represents an essential component of the early stage of infant feeding, whether it involves the mother's expressed breast or a suitable pacifier. It further contributes to physiological stability, enhances nutrient absorption and $\mathrm{Cl}$ track functioning. These facilitate the process of transition from tube to oral feeding due the acceleration of maturation (7,30,31). In the 2016 Cochrane meta-analysis involving infants born $<37$ weeks of $\mathrm{GA}$, it was shown that NNS shortened the transition from gavage to FOF by 5.51 days and reduced hospitalization by 4.59 days (7). Noori et al. (32), in their study with infants born at 26-34 weeks of GA, showed that NNS via the mother's finger sped up time to FOF and reduced hospitalization in intervention groups in comparison to control groups; $22 \pm 14.51$ vs $30.05 \pm 18.58$ days, and $31.26 \pm 16.89$ vs $41.82 \pm 23.07$ days, respectively. The findings of our study in infants born at $<32$ weeks of GA showed that KMC and NNS provision within the first week was associated with a 7.1-fold increased likelihood of transition to FOF within 20 days, and similar to the previous study by Noori et al. (32), these infants were discharged from hospital within 30 days.

Not surprisingly, the findings of this study are in agreement with the existing evidence indicating that younger neonates with comorbidities require additional time to consistently exhibit oral feeding skills. On the other hand, it is interesting to observe that until now, few studies have explored the effects of comorbid conditions on the feeding process among VPT infants $(6,19,23,24)$. The present study appears to be the first to evaluate and demonstrate the effects of KMC and NNS provision on time to FOF. However, the current study has several limitations. This was a single center study with a retrospective design, which aimed at examining well-known factors in neonates. There may be other variables not evaluated in this study that possibly affect FOF. Also, it is known that the type/ mode of feeding has an impact on the feeding process. Unfortunately, due to the fact that the majority of our study subjects received nutritional support with formula and/or bottles, it was not possible to determine the type/mode of feeding which has a possible influence on the time to achieving FOF.

\section{Conclusion}

In conclusion, this is one of the few studies to demonstrate the timing of transition to FOF and its determinants in VPT infants. Further multicenter studies with further infants are required to provide guidance on interventions aimed at shortening time to FOF as well as to provide more insights into the roles of KMC, NNS and breastfeeding on the VPT population. 
Acknowledgement: Thanks to Prof. Canan Baydemir, Department of Biostatistics and Medical Informatics, Kocaeli University Faculty of Medicine, for her help in the statistical analysis.

\section{Ethics}

Ethics Committee Approval: The study protocol was approved by the University of Health Sciences Turkey, Kocaeli Derince Training and Research Hospital, Clinical Research ethics committee (no: 2020/72).

Informed Consent: Retrospective the study.

Peer-review: Externally peer-reviewed.

\section{Authorship Contributions}

Design: I.E., A.G., Data Collection: I.E., A.G., Analysis or interpretation: I.E., A.G., Literature Research: Writing: İ.E., A.G.

Conflict of Interest: The authors declared no conflict of interest.

Financial Disclosure: The authors declared that this study received no financial support.

\section{References}

1. American Academy of Pediatrics, Committee on Fetus and Newborn. Hospital discharge of the high risk neonate: proposed guidelines. Pediatrics 2008; 122:1119-26.

2. Stade B, Bishop C. A semidemand feeding protocol reduced time to full oral feeding in healthy preterm infants. Evid Based Nurs. 2002; 5:74

3. Lubbe W. Clinicians guide for cue-based transition to oral feeding in preterm infants: An easy-to-use clinical guide. I Eval Clin Pract 2018; 24:80-8

4. Sadler TW. Special embryology: respiratory system and digestive system. In: Sadler TW (ed). Langman's Medical Embryology,7th ed. Baltimore, MD, Williams and Wilkin: MD, 1995; 232-71.

5. Browne JV, Ross ES. Eating as a neurodevelopmental process for high-risk newborns. Clin Perinatol 2011; 38:731-43.

6. Jadcherla SR, Wang M, Vijayapal AS, Leuthner SR. Impact of prematurity and co-morbidities on feeding milestones in neonates: a retrospective study. J Perinatol 2010; 30:201-8.

7. Foster JP, Psaila K, Patterson T. Non-nutritive sucking for increasing physiologic stability and nutrition in preterm infants. Cochrane Database Syst Rev 2016; 10:CD001071.

8. Kültürsay N, Bilgen H, Türkyılmaz C. Turkish Neonatal Society guideline on enteral feeding of the preterm infant. Turk Pediatri Ars 2018; 53(Suppl 1):S109-18

9. Hay WW Jr. Strategies for feeding the preterm infant. Neonatology 2008; 94:245.

10. Fenton TR, Kim JH. A systematic review and meta-analysis to revise the Fenton growth chart for preterm infants. BMC Pediatr 2013; 13:59.
11. Dutta $S$, Singh $B$, Chessell $L$, et al. Guidelines for feeding very low birth weight infants. Nutrients 2015; 7:423-42.

12. Schanler RJ. Approach to enteral nutrition in the premature infant. In Up To Date, Abrams SA, Hoppins AG (Eds), Up To Date 2017. Available from: https://www.uptodate.com/contents/ approach-to-enteral-nutrition-in-the-premature- infant -

13. Parker L, Torrazza RM, Li Y, Talaga E, Shuster J, Neu J. Aspiration and evaluation of gastric residuals in the neonatal intensive care unit: state of the science. J Perinat Neonatal Nurs 2015; 29:51-9.

14. Berman L, Moss RL. Necrotizing enterocolitis: an update. Semin Fetal Neonatal Med 2011; 16:145-50.

15. Heuchan AM, Clyman RI. Managing the patent ductus arteriosus: current treatment options. Arch Dis Child Fetal Neonatal Ed 2014; 99:F431-6. doi: 10.1136/archdischild-2014-306176.

16. Dong $Y$, Speer CP. Late-onset neonatal sepsis: recent developments. Arch Dis Child Fetal Neonatal Ed 2015; 100:F25763.

17. Higgins RD, Jobe AH, Koso-Thomas M, et al. Bronchopulmonary dysplasia: executive summary of a workshop. I Pediatr 2018: 197:300-8.

18. Härtel C, Haase B, Browning-Carmo K, et al. Does the enteral feeding advancement affect short-term outcomes in very low birth weight infants? J Pediatr Gastroenterol Nutr 2009; 48:46470

19. Brun G, Fischer Fumeaux C), Giannoni E, Bickle Graz M. Factors associated with postmenstrual age at full oral feeding in very preterm infants. PLoS One 2020; 15:e0241769.

20. Kish MZ. Oral feeding readiness in preterm infants: a concept analysis. Adv Neonatal Care 2013; 13:230-7.

21. McGrath JM, Braescu AVB. State of the science: feeding readiness in the preterm infant. J Perinat Neonatal Nurs 2004; 18:353-68.

22. Barlow S, Finan D, Lee I, Chu S. Synthetic orocutaneous stimulation entrains preterm infants with feeding difficulties to suck. ) Perinatol 2008; 28:541-48.

23. Giannì $M L$, Sannino $P$, Bezze $E$, et al. Effect of co-morbidities on the development of oral feeding ability in pre-term infants: a retrospective study. Sci Rep 2015; 5:16603.

24. Hwang YS, Ma MC, Tseng YM, Tsai WH. Associations among perinatal factors and age of achievement of full oral feeding in very preterm infants. Pediatr Neonatol 2013; 54:309-14.

25. Dodrill $\mathrm{P}, \mathrm{McM}$ ahon $\mathrm{S}$, Ward E, Weir K, Donovan T, Riddle B. Long-term oral sensitivity and feeding skills of low-risk preterm infants. Early Hum Dev 2004; 76:23-37.

26. Neubauer $V$, Junker $D$, Griesmaier $E$, Schocke $M$, KiechlKohlendorfer $\mathrm{U}$. Bronchopulmonary dysplasia is associated with delayed structural brain maturation in preterm infants. Neonatology 2015; 107:179-84.

27. Conde-Agudelo A, Belizán JM, Diaz-Rossello J. Kangaroo mother care to reduce morbidity and mortality in low birth weight infants. Cochrane Database Syst Rev 2011; 3:CD002771.

28. Er I. Perinatal factors that influence early transition to oral feeding in preterm infants: a retrospective study. 1. International Congress of "Breastfeeding Reality", September 30-October 3, 2019, Ankara. 
29. Nijhuis JG. Fetal behavior. Neurobiol Aging 2003; 24(Suppl 1):S41-6; discussion S47-9, S51-2.

30. Bingham PM, Ashikaga T, Abbasi S. Prospective study of nonnutritive sucking and feeding skills in premature infants. Arch Dis Child Fetal Neonatal Ed 2010; 95:F194-200.

31. Bache M, Pizon E, Jacobs J, Vaillant M, Lecomte A. Effects of prefeeding oral stimulation on oral feeding in preterm infants: a randomized clinical trial. Early Hum Dev 2014; 90:125-9.
32. Noori F, Nariman S, Rahmiaan H, Sadat-Hoseini AS, Ravarian A. The effect of non-nutritive sucking by mothers on full oral feeding attainment in preterm neonates, I Compr Ped 2018; 9:e59657. 